\title{
Organization and Technology Management Issues of Joint European Projects in a Factory and in a Research Institute
}

\author{
George L. Kovács*, János Nacsa*, \\ István Lepsényi*** \\ * CIM Research Laboratory, Computer and \\ Automation Research Institute, Hungarian \\ Academy of Sciences, 1518, Budapest, POB \\ 63, Hungary \\ ** Knorr Bremse Brake System, Ltd., 6000 \\ Kecskemét, $P O B$ 97, Hungary
}

The paper summarizes three EC projects where Hungarian companies were involved. In the frame of the EC-Program COPERNICUS a CAD-Based Programming System for arc welding robots has been developed by a consortium with German and Hungarian participants. The basic goal of the system is to assist robotized arc welding in Small and Medium Size Enterprises (SME) mostly for small and medium lot sizes. PROARC which will be introduced in more detailed is implemented in AutoCAD, and the system was integrated into an open CIM environment.

Knorr Bremse is running two EC supported PHARE projects. One of them takes into account the ISO 9001/2 standard for measurement and calibration increase the market shares. The other project is a strategic software development to avoid the loss of stability and loss of trace of trucks by using pattern recognition and up-to-date control means to manage braking properly.

Keywords: European cooperation, PHARE, COPERNICUS, industry-university cooperation, robots, arc-welding, OSI, ISO 9000,



George L. Kovács was born in Budapest, 1943. He got his diploma (MS), Ph.D. and habilitation (Prof. degree) at the Technical University of Budapest in 1966, 1978 and 1995 respectively. Since 1966 he is an employee of the Computer and Automation Research Institute of the Hungarian Academy of Sciences, recently he is the head of the CIM Research Laboratory. His professional interest is manufacturing automation, application of intelligent tools in the design, scheduling and control of flexible manufacturing systems. He has been visiting the USA, Russia, Germany, Mexico and Italy as visiting researcher or as visiting professor for longer terms. He is a member of IEEE, and of different IFAC committees, he is the Hungarian Representative of IFIP in its TC5.

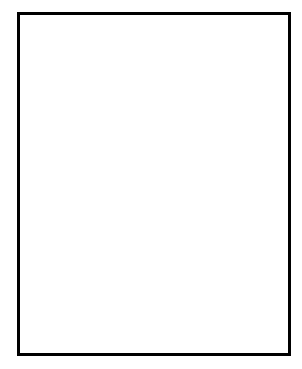

János Nacsa was graduated in 1987 at the Technical University of Budapest in 1987. Since that time he is working for the Computer and Automation Research Institute of the Hungarian Academy of Sciences as research associate. He was involved in many different projects in the field of manufacturing automation, and was specially responsible for the PROARC project. His research interest is industrial communication, application of distributed artificial intelligence in manufacturing, open controller architectures

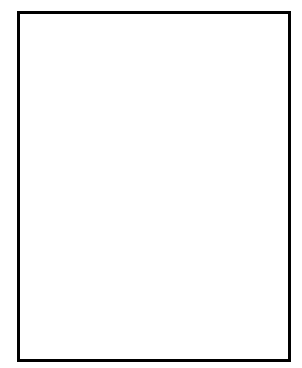

István Lepsényi joined IKARUS, the bus giant after graduating at the Technical University of Budapest in 1974. He has introduced robots, manufacturing cells and flexible manufacturing systems in the production, the first CAD system in development. From 1986 he was the technical director of IKARUS.

In 1990 he founded AUTOKONSZERN, a consortium aiming car production in Hungary. As a result Suzuki Hungary Co. was founded in 1991 - as the only Japanese car manufacturing plant in Europe and he became the first president of the company. He joined Knorr Bremse, the leading company in pneumatic brakes in 1995. He got Széchenyi prize in 1996.

\section{Introduction}

In this paper we shall discuss two types of European joint R/D activities: 
- a joint European COPERNICUS project, and the participation of the Computer and Automation Research Institute (CARI) in the project,

- two PHARE supported projects of the factory Knorr-Bremse. One of them is a quality assurance project, while the other one is an $\mathrm{R} \& \mathrm{D}$ project with the participation of CARI and the Technical University of Budapest.

First we are going to give some important technical details on the projects to see the innovation, the quality increasing effects of the new products produced by the development. Then we shall give a short analysis of the lifecycles of these projects giving information on the following main issues:

- proposals, application procedures and partners' selection

- contracts with the European Community

- project development

- advantages for the participating and for other future partners, including consumers.

Finally some conclusions and suggestions close the paper.

Because of the nature of the projects the COPERNICUS project will be given in details, while only the main issues of the PHARE projects will be discussed.

\section{The projects}

\subsection{The PROARC COPERNICUS Project}

A large number of industrial robots has been established in the field of arc welding over the last twenty years. The reliability of robots, their use in more than two shifts and the reproducible welding quality are arguments for the use of this technology. However, the application of arc welding robots is mostly limited to the production of big lot sizes. The classical way to program a robot is still the teach-in method in which the robot has to be led to the various points, which afterward will be connected by a manually generated program. This programming method is highly insufficient and not economical for small or medium lot sizes. Therefore off-lineprogramming systems have been developed to decouple the process of program generation from the actual robot in the workcell.

Many different programming systems are available on the market which meet the different requirements of the users. At the beginning of the PROARC project more than ten systems were analyzed [2]. Some samples are given below:

The Windows based WORKSPACE system from Robot Simulations [9], GRASP [5] is a 3D graphical simulation tool with off-line programming features including a welding assist menu. AnySIM [4] is a general simulation tool which has a basic module and special extensions for simulating $\mathrm{NC}$ machines, human resources, etc. Finally, the ACT WELD from Aleph Technologies is the only PC-based system, which especially supports welding functionalities [1]. is the welding extension of ACT. ACT is a CAD/CAM robot software with extra open $\mathrm{C}$ interface for programming that is not usual for such systems. It has a 3D tool for modeling a complete workcell with visualization features. It is open for different CAD systems. It is a powerful system providing not only automatic generation of the welding paths and the corresponding technology data, but also a collision free path planner for the robot's movements. The system is executable on PCs (DOS) as well as on Workstations (UNIX) and besides the ACT WELD module further modules e.g. for Autonomous Vehicles, etc. are available. However, as none of the previous systems, neither ACT WELD uses common commercially available software tools such as the graphics modeller ACIS or the development platform AutoCAD. This is a major drawback, since the reimplementation and maintenance of basic functionalities need much more efforts (man power and development time) as when common modules 
are used. Another important limit of ACT is that it supports only the low level controller (called KALI) of the developer company.

The systems differ from simple textual editors up to high performance 3D graphical environments including modules to enhance the use of the systems functionalities and the speed of program generation. But most of the functions are distributed over the different systems and none of the systems are especially designed for the use in SMEs for welding applications.

PROARC is capable of simulating [11], generating and reusing entire or partial welding sequences for robot program generation. The typical cell layout consists of a robot and a positioner. Torch cleaning and changing stations are also supported and the corresponding motions are automatically included in the welding sequence. The workpieces can have single or multiple seams and can be shifted individually. Technology macros have also been defined for fillet welds and V-type welds. Finally, PROARC is capable of importing different CAD formats as well as communicating with various robot controllers via an MMS (Manufacturing Message Specification) interface [10]. We concluded that the commercially available offline robot programming systems are either too expensive or cannot provide all required functionalities.

In the following the approach of the PROARC system will be discussed. First, the goals and the structure of the system are shown where the program generation and the communication modules are introduced in more details [6]. Finally, in chapter 6 the demonstration environment and its components will be explained shortly.

\subsubsection{PROARC objectives}

The above examination of the available programming systems allowed to the draw some conclusions which were useful for the development of the PROARC system.
The target group are clearly SME companies, which consequently cannot afford so high investment costs as large companies do. The costs of the required computer type, additional hardware and software had to be kept low in order to encourage the companies to invest in new technologies. Personal computers and standard software are important preconditions for this goal. Furthermore, the use of well known software tools for the realization of a new system enables the user to work with the new system effectively after a very short time of practicing. The user will accept the system faster and the productivity will raise with the user's motivation.

Out of this motivation the AutoCAD system has been chosen as the development platform for the PROARC system. It meets the precondition of a frequently used software in SMEs and it provides the required functionalities to develop a user friendly programming environment. E.g. the user should be freed from complicated input sequences and should be able to provide his knowledge of a welding expert for the system in a simple way. Thus, the PROARC system is able to concentrate on the application specific features. Some basic capabilities of the system are similar to those of ACT WELD. However, the major difference is the flexibility of the seam's programming in PROARC. No system is known, which allows a flexible restructuring of the welding sequence and the unconstrained back (modeling) and forth (simulation) stepping within a single programming session. The basic requirements of the PROARC system defined by the consortium were the following, grouped in three categories:

\section{Modeling}

- support of standard CAD interfaces (IGES, DXF);

- 3D solid modeling of the entire workcell, including robot, positioner, torchstation, and cleaning device; 
- support of collision checking during simulation;

\section{Programming}

- support of Fillet and Butt welds, multiple layers build from straight and circular weldlines;

- automatic generation of welding paths including the appropriate welding data;

- unconstrained programming of welding sequences;

- effective access to welding entities;

\section{Program generation and communication to} the robot

- support of a common interface between the programming and program generation part of the system to provide the use of different robot languages;

- the DNC connection support of an MMS interface between the programming system and the robot to provide more control over the welding process for the user;

\subsubsection{PROARC System architecture}

The system can be divided into different functional modules: Modeling, Welding Planning, Program Generation and Communication. In this division of the system the different phases of an off-line programming session are mapped. However, the underlying structure is not that separable, especially not for the first two modules.

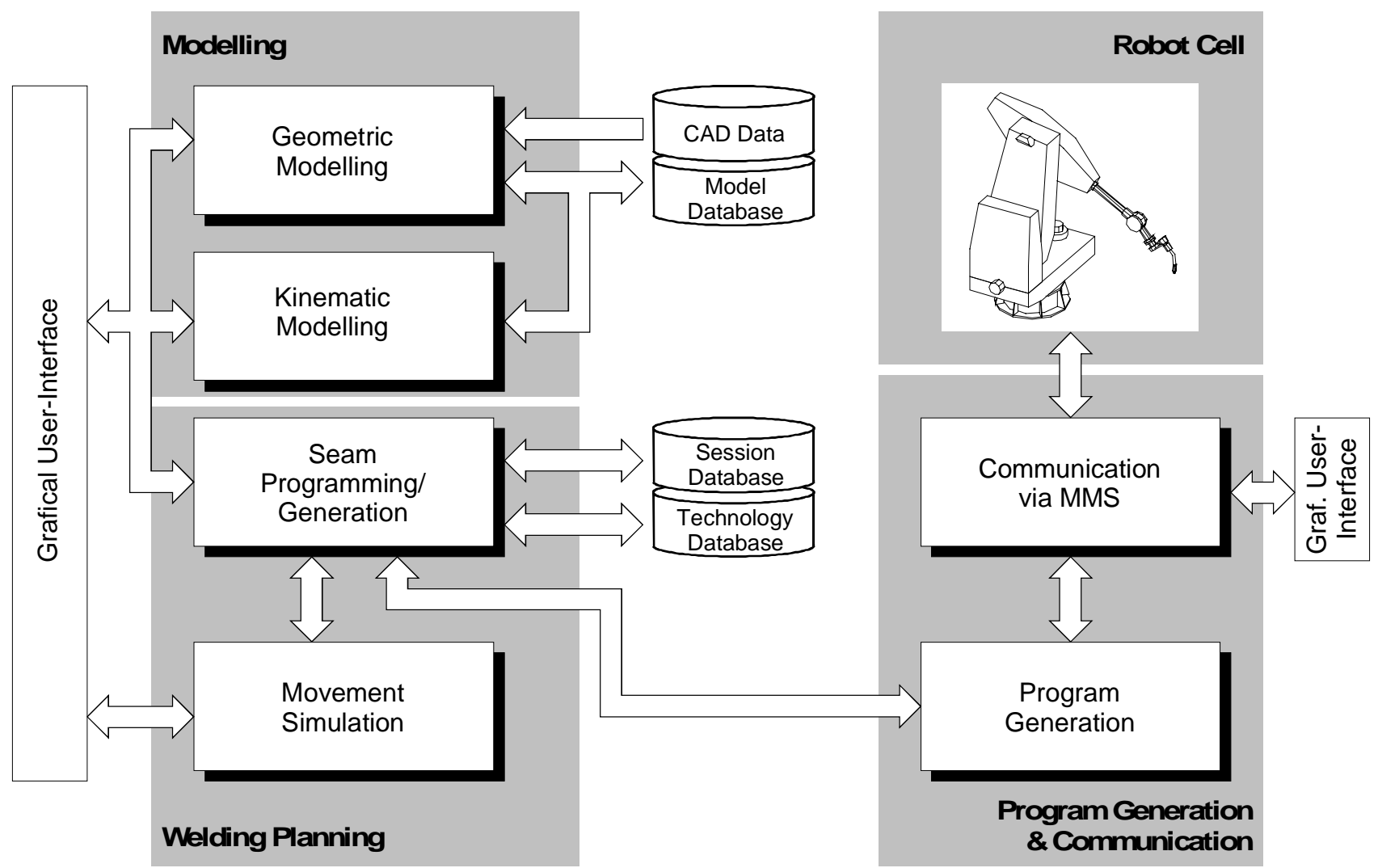

Figure 1: Principle software structure of the system

For the development of the system's kernel, an object oriented approach has been taken. Object oriented software development has a number of advances versus the conventional top-down approach. Some of them are modularity, information hiding, polymorphism, which often result in a better ability for the maintenance and reusability of 
the software. In this project the Booch notation [3], has been chosen for the development. It can be used during the entire cycle of software development (analysis phase, design phase, and implementation phase). These three phases were developed in an iterative way using the concurrent engineering methodology. That means, that some parts of the software might be in the state of implementation while some other parts might just have been analyzed. Thus, taking an other view of the system leads to Fig. 1. which describes the software structure of the system.

This was the motivation for, on the one hand, the modular design of the problem domain classes and, on the other hand, the introduction of a further tool to implement the user interface. This tool, the Microsoft Foundation Classes (MFC) is supplied by the Visual C++ compiler and enables the programmer to create very efficiently a Windows driven application framework. Thus, the connection to AutoCAD is reduced to the management of the graphical representations of different entities. This connection is established by a set of classes specific for the AutoCAD software. Finally, for the persistent storage of the system's runtime objects, serialization functionalities are used, which again are supplied by the MFC.

The Modeling Module of the system provides the model of the welding environment to test for possible collisions. There are two fundamental components of the welding environment: the workpiece (with fixtures), and the entire workcell. cleaning/changing). The model used in the PROARC project also includes the obstacles (e.g. the ceiling or the floor) as well. The robot and the positioner are modeled as a kinematics machine with joints and links [7]. The workpiece is modeled as a whole. This means, that the geometry of the workpiece is modeled in its welded state when all of the parts are joined together. In this case the designation of the seams is done by picking edges on the surface of the workpiece. Presently three different torch types with three different geometries are supported in the PROARC system.

In the Welding Planning Module the user is able to program seams on the $3 \mathrm{D}$ model of the workpiece interactively. In contrary to existing off-line programming systems for welding our system does not only provide the processing of single seams, but it allows the user to access each layer of a seam individually. Furthermore, the user is able to define his/her own sequencing of the layers and thus the welding experience is used in this phase of the programming. Macros have been introduced, which enable a fast and efficient posing of the welding torch and association of technology information to the seams. In all states of the program it is possible to simulate the movements of the robot and the optional positioner. Thus, the user is able to jump back and forth between the programming and the visualization phases. This gives a maximum controllability of the welding process to the user.

The programmed welding session will finally be translated into the robot language and then downloaded to the robot. This is supported by the Program Generation/Communication Module. The interface to the Program Generation Module is defined in STEP standard „EXPRESS“ language. Since no predefined or standardized interfaces for the exchange of welding data exists, new STEP entities (exchange datatypes) were introduced. These entities apply to the demand to be that general, that they cover the capabilities of a large number of robot languages.

Presently two torch specific operations were introduced: torch changing and torch cleaning. There are welding attributes belonging to a complete seam which is one welding operation:

In order to possess a maximum control over the robot while working at the off-line 
programming system, an MMS interface has been implemented. In a first development step mainly the basic communication functionalities are supported. However, beside this MMS connection a common DNC connection has also been established. This additionally enables the basic communication to most robot controller types.

\subsubsection{The PROARC Robot Program Generation Module}

The main function of the Program Generation module is the compilation of the STEP based robot movement description including the welding features to the actual arc welding robot language with a format, which can be directly downloaded into the robot controller. This means, that the module has strong dependencies to the actual robot environment and has to be modified, if applied to a robot controller. However, the internal structure of the module supports this programming effort. So this module is allow implemented in an independent program to the exchange of it with a similar one if another application uses another environment.

The off-line-programming session defines the entire sequence of movements for the welding of a workpiece. However, there will be deviations between the generated positions and the real positions e.g. due to calibration errors or inaccuracies of the real workpiece. Therefore a set of search movements has been specified, which determine the real position of the workpiece by using a tactile sensor. These movements are defined for different workpiece geometries.

The robot depending parts of the program generation task can be summarized in three points. The first one is the language of the robot, the second is the format of the up- and downloading and the third one is the robot interface of the welding source, how the features of the welding can be reached from the robot.

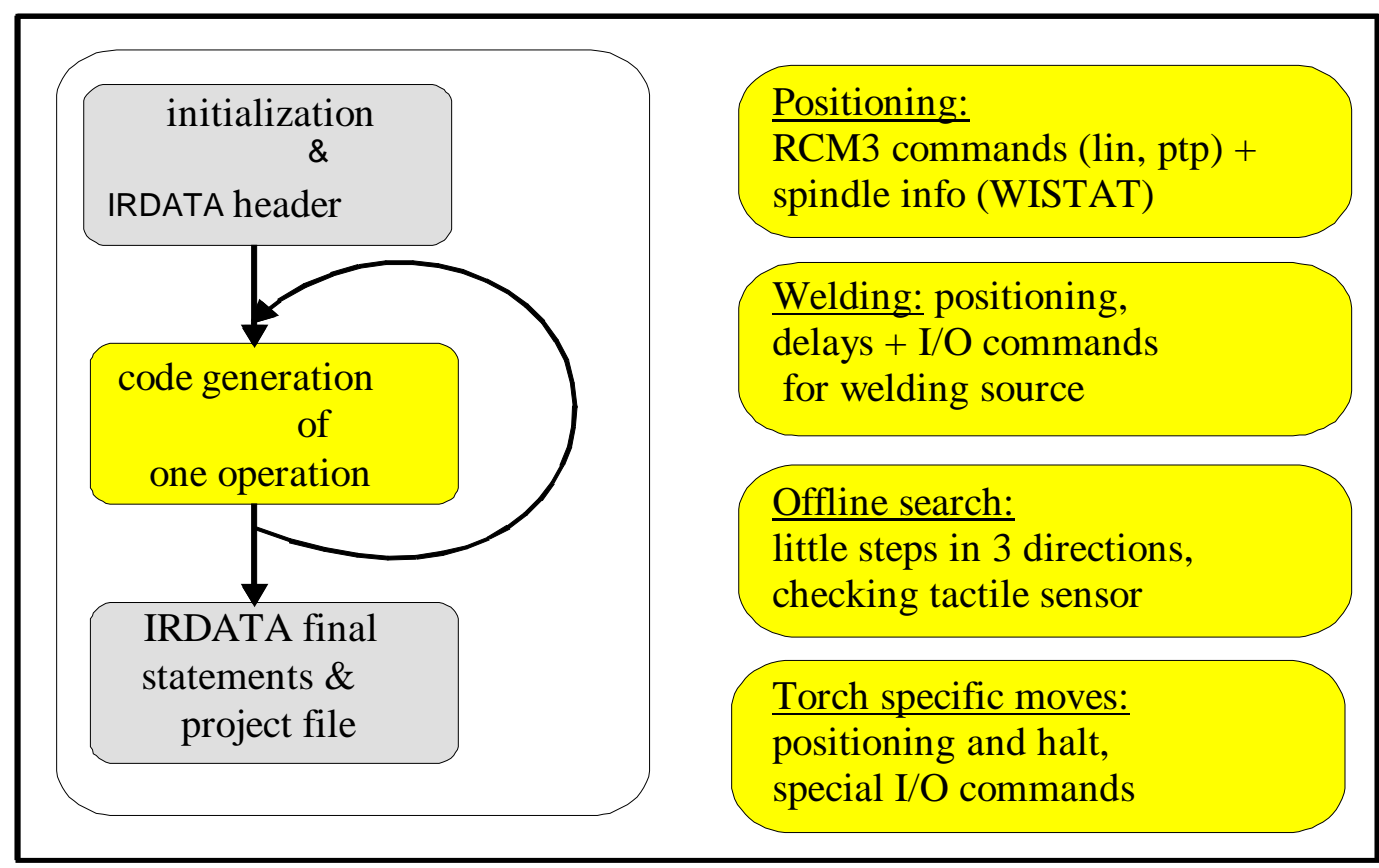

Figure 2: Creation mechanism in the program generation module

The creation mechanism (Fig. 2.) of the robot programs were developed in step by step algorithms splitting up the different operations. Each operation is described by objects which consist of STEP program statements together with given robot 
programming language code parts. Some mappings have, of course, variable parts (e.g. coordinate values, delays, wirespeed). Some additional features of the program generation module were also defined to give some comfort to the user. They are general features, independent from the given environment and can be used in other versions of the program for other robots, too.

All the parameters of the program generation are stored in a so called 'task' file. The user is able to generate it automatically and all the modifications are written directly to the file. The task file contains not only the input and the output of the program generation (STEP file and robot program), but also special switches (for example welding on, search on, etc.).

Different types of robot programs can be generated from the same source STEP file. The 'welding off' switch allows to check the exact path of the robot during the real movement without the welding itself. Three execution types of searches are defined. Programs can be generated with skipping searches ('search off'), with checking only the correct positions ('skip seam group on') and using the 'grouping' of the search and welding moves the robot can change its path according to the result of the searches ("skip seam group off'). It is possible to set the accuracy of the search.

The user can take a look at the files within the program, but the direct editing of them (either the STEP or the robot program files) are not allowed. It was decided because of safety reasons, but it can be changed easily if it is requested.

The modules of Program Generation shown above in Fig. 3. are linked together to get a single executable (EXE) file, but the modification of them to a single dynamic link library (DLL) would be easily possible. In the following the modules' structure and their tasks will shortly be described.

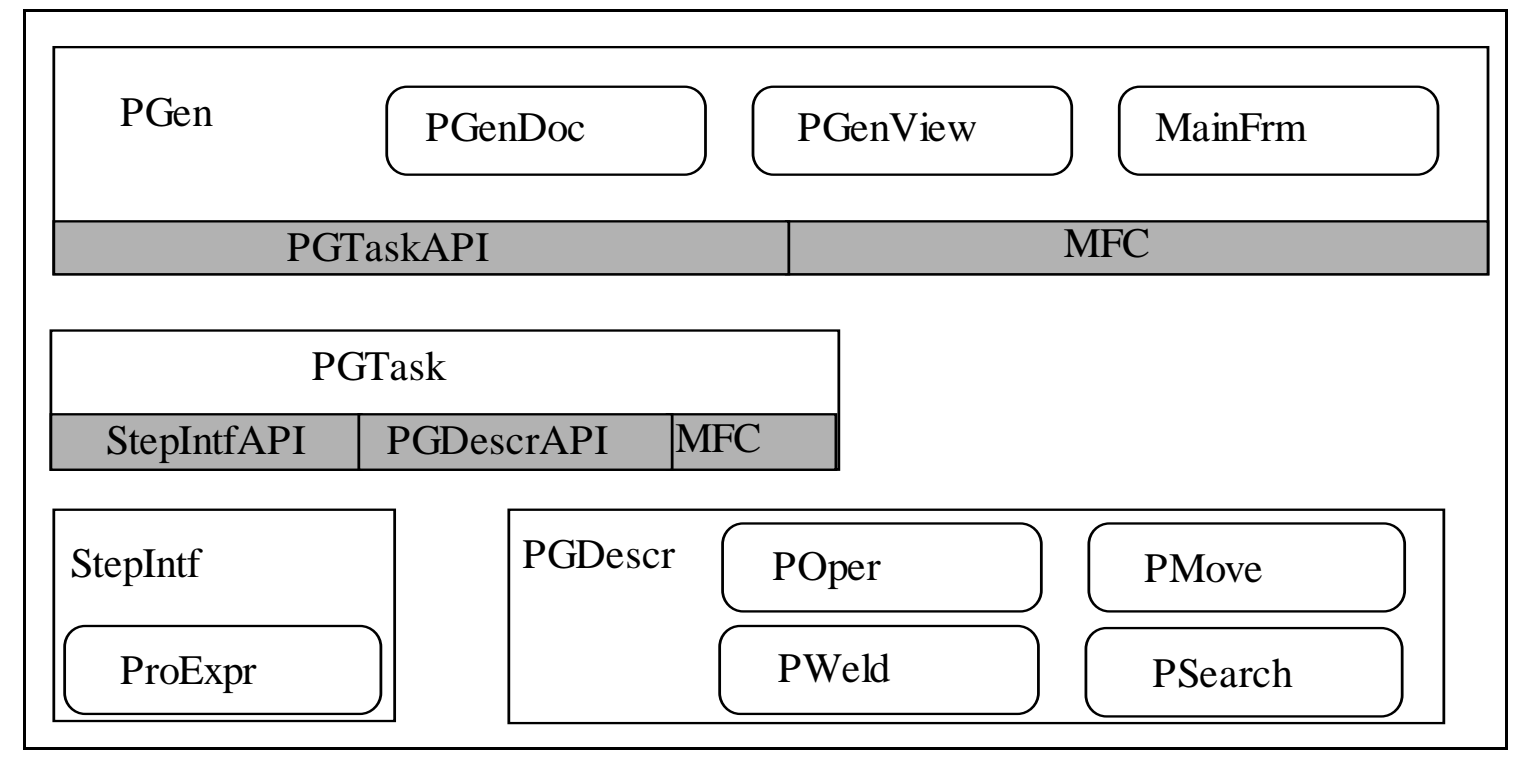

Figure 3: Software modules of the program generation

\section{PGen}

This part handles the user interface of the Program Generation. The frame of this module and the three related ones was generated using the Application Studio tool of MFC. All the
Dialog Boxes and one Menu was defined within the tool. The default Dialogs are used for file opening and saving and some others allow to set different values (e.g. (re)edit the task file data, or change some search options). 
As an extra tool with the simplest 'view' technology of MFC it is possible to look at any STEP or robot program file.

\section{PGTask}

It manages all the operations of the different files (STEP, robot program and task) in three classes. The program generation function is built up from an ordered set of these operations. This set contains general file management and special operations dedicated to a given class (e.g. load non data parts of the STEP file, update the task file with the new search options). This module contains an additional class which describes the actual robot (e.g. existence of the sensors, I/O channels belonging to the different welding features).

\section{StepIntf}

This module translates the entity based STEP description into an internal representation of the program and checks the different errors. It has a special structure which is independent from the PROARC STEP specification, so it could be used as a preprocessor of any STEP based files. The PROARC specific definition is given in the 'proexpr' part of the module.

\section{PGDescr}

The operations of the classes of these modules are doing the real program generation work. The classes are used to define how the different elements of the robot program should be generated. They were built up to give a robot independent structure, where only the contents of the classes should be rewritten for another robot program. The present code pieces within the operations are developed specially for the demonstration environment. Pmove is responsible for the elementary movements, Pweld generates the welding specific parts, Psearch creates search subroutines within the robot code and Poper has different routines belonging to every operation defined in the STEP specification. There are additional parts within PGDescr generating the frame of the robot program (header and footer parts of the robot program).

\subsubsection{The PROARC Communication Module}

Beside the programming of welding tasks, another common problem is the communication between the off-line programming system and the robot itself. The following main set of functions may be used during the remote control session in increasing complexity:

- program download/upload, delete; remote start/stop,

- determination of robot status (idle, loading, executing, etc.); read/write different variables

- create/delete variables; performing event handling

- multi-access by multiple hosts

Most robot controllers provide only optional DNC connections via serial line and typically allow only a limited number of operations via that channel (e.g. download/upload). This serial connection has two main disadvantages:

- It obviously means a point to point connection; so it is not possible to access or monitor the robot from multiple computers.

- The speed of the communication is low.

Users wish to quickly connect robots to their network in a 'client-server based' manner. They prefer low-cost and vendor independent solutions, which are consequently standard based networks instead of proprietary ones of a single vendor. These connections mean very different remote interfaces of the robots. As an alternative, the introduction of a standard based network solution such as MMS via Ethernet offers good capabilities and with the implementation of special Network Interface Units (NIU) to the robots even the multi-access and the common interface problems can be solved. 
So looking for a general interface for the PROARC system towards the robot controllers, the MMS (Manufacturing System Specification) protocol and interface was chosen. It provides all the functionalities discussed above. More and more robot vendors offer such interface, and there are proven applications where MMS interface was implemented for a non MMS robot controller successfully [12]. As the robot companion standard of the MMS exists it supports the application developers to build general tools to be able to communicate with many different types of robots.

The functions are used mainly by the Domain and Program Invocation (PI) Services of the MMS. The PI services allow the robot control functions: start, stop, continue of the robot program and enable the user to choose between different programs on the controller storage medium (create PI). The domain services provide the up- and download of selected programs.

This module is a Client on the MMS network exchanging messages with the robot's MMS interface. Because of the present situation, that none of the industrial controller vendors support the extensions of MMS, which is necessary for the Robot Companion Standard, its original model had to be redesigned in order to make it applicable to the real cell. The MMS Client based on a Virtual Manufacturing Device (VMD) model of the robot was specified to provide a robot independent general 'Robot VMD'. Additional MMS objects were introduced to get information about the status of the welding equipment.

The connection can be established with an initiation message and closed with a conclude message. Both communicating nodes, the PROARC station (Communication module) and the robot (more precisely its NIU) can skip the connection with an abort message.

Some status actions were built in to allow the user to get information continuously from the robot. It was implemented because in the networking point of view the robot is not active, so all the information is coming from it via message poling. It is important, because without it, the Communication Module will not know if the robot program ends, if manual torch cleaning or changing is necessary or not.

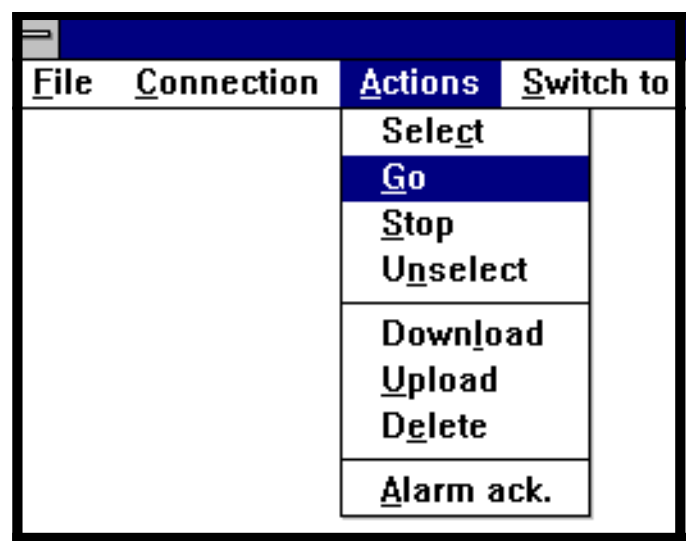

Figure 4: The Actions menu in the Communication Module

The menu list in Fig. 4. shows the actions the user can activate to work with the robot. The first group of the actions supports the direct remote control operations (using the MMS Program Invocation services), the second group manages the robot programs within the controller (MMS Domain Management services). There is a further possibility (via an MMS Variable called R_ALARM) to acknowledge the alarms of the robot controller (e.g. Transmission Error) and the warning messages programmed in the robot code (e.g. Change the torch or No workpiece found).

If the user wants to work here also with the task files created in the Program Generation module, then he/she gets the download file directly from the task file data. If an earlier uploaded version of the same robot program exists, than a warning message forces the user to choose which one to download. Similar warning will be seen if the user wants to upload the same robot program again. In one 
control session only one open task file is allowed.

The Communication Module was designed to be an independent program running under MS-Windows. It is also based on the MFC objects. The file management is also supported by the MFC. For the MMS communication the most commonly spread out Application Programming Interface (API), the MMSEASE is used [8].

The modules of the Communication are also linked together to have a single executable (EXE) file. The software structure is rather simple: the user interface and file management parts (Rcom) are connected via a general link API to the MMS-EASE parts of the program. The structure of Rcom is very similar to Pgen of the Program Generation, of course the functionality is different.

\section{Link}

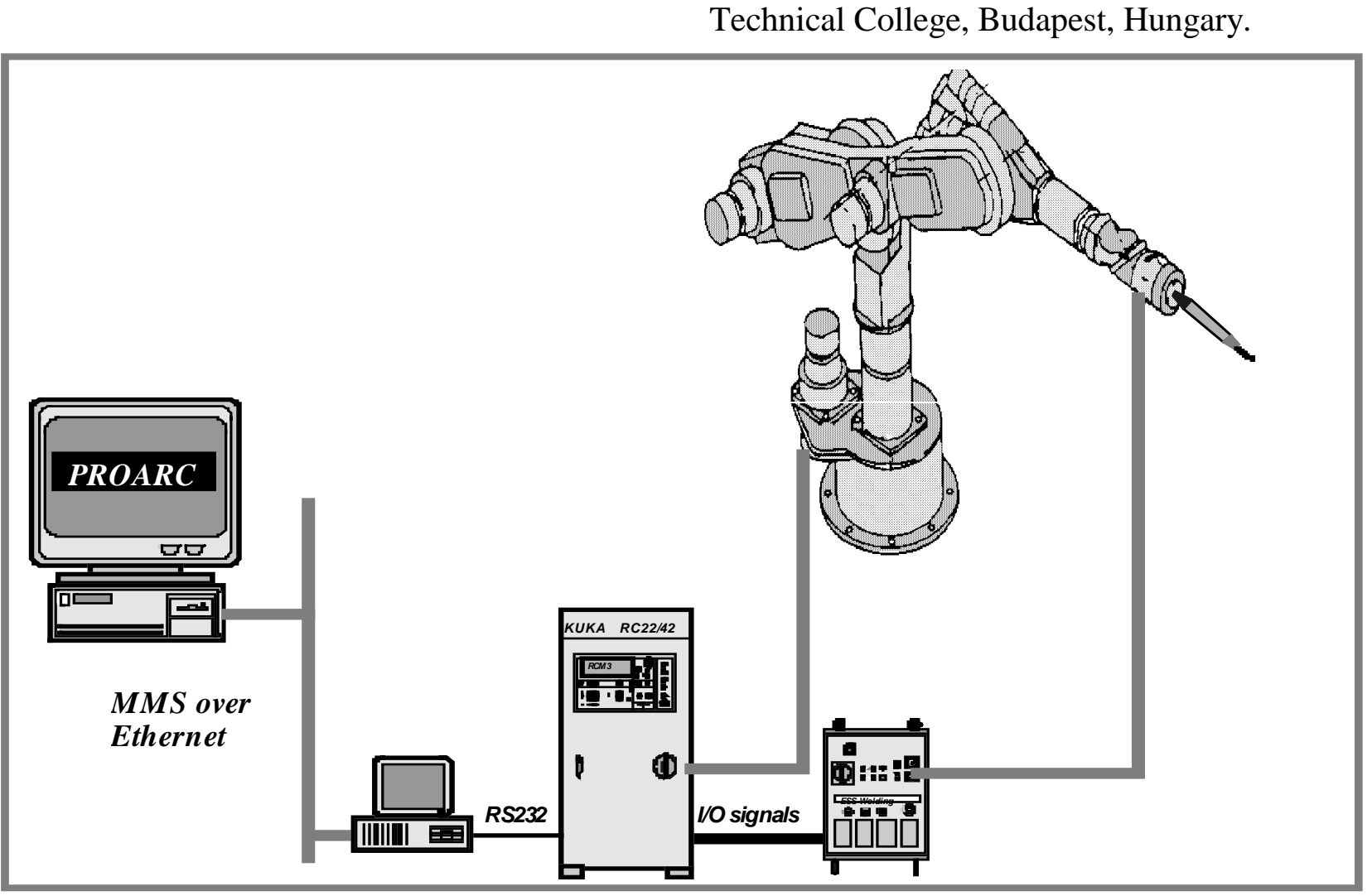

Figure 5: Final demonstration environment of the PROARC project

\subsubsection{The PROARC Demonstration Environment}

For the demonstration of the project's results an arc welding robot was needed that supplies a MMS interface to be able to communicate with the PROARC system. It was not possible to find such a robot in Hungary, so it was decided to develop a MAP interface to an arc welding robot that has a serial DNC connection. An RCM3 KUKA robot was chosen with an ESS welding equipment (Fig. 5.) in the workshop of the Automation Institute of the Kando Kálmán Technical College, Budapest, Hungary.
The link API was developed in a previous calls, the MapConf the confirmations and the MapMust contains the further necessary elements required by the MMS-EASE API (e.g. initialization, error handling).

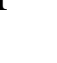
project, and gives a general interface for part the MapReq collects the network request 
A NIU module was developed, which connects the RCM3 robot to the MMS network. This NIU is an MMS Server communicating with the MMS Client of the Communication Module. It sends the MMS messages to the RCM3 via its LSV2 based DNC protocol. The robot depending part, the functional mapping of the general VMD model and the given demo environment, was also developed for the NIU.

The demonstration environment of the project did not support all the features provided by PROARC. For instance, only one torch (type 0 degree) was available and some welding features were not used (e.g. arc sensor). Only a simple tactile sensor was implemented to be able to demonstrate the different search modes and sequences.

\subsubsection{Some Conclusions of the PROARC Project}

It has been shown, that the commercially available off-line programming systems for welding applications do not cover the needs of SMEs with small batch sizes and with one-ofa-kind production to a sufficient extent. Factors such as low costs, simple usability, and a well known software basis on the one hand and effective functionalities, reusability of welding programs, and an enhanced communication with the robot on the other hand have strongly influenced the development of the proposed PROARC system.

The joint COPERNICUS project was a success for both the Western (WZL, RWTH Aachen) and the Eastern (University of Veszprém and Computer and Automation Institute, Budapest) partners, as we learnt to work together effectively based on personal discussions and electronic communications, using equivalent software and hardware tools, influenced by the conclusions of the review meetings organized by the EU.

We had to solve several interesting problems during the development, which might be useful independently of PROARC, too. For example building the robot inverse kinematics into the AutoCAD, implementing our general robot link interface into the communication module etc.

As a final result of the project we got a good prototype solution, which should and could be transformed to a useful product if some more further cooperation and financing could be managed to solve some more open problems, as for example a more sophisticated simulation of the robot movements in AutoCAD.

\subsection{Quality: the first Knorr-Bremse Project}

Quality is the main issue of the PHARE supported project of Knorr-Bremse. The quality of products has to be increased to increase market shares, to have better position in the world wide competition. The factory has a PPM of 800.000 recently. It should be increased to 1 million using the new test facilities which are under development today. The main purpose of the factory is to have an accredited test laboratory establishment to be used as internal quality assurance center and to be used to test all equipment of business partners delivered to Knorr Bremse Nowadays this type of cooperation between business partners delivering parts or assemblies to each other is a must. This is the only way that a uniformly high quality of the end products can be achieved. The recent ISO 9000 compliance is planned to be increased to achieve QS-9000 within some years, as it is already the quality requirement of the next century.

Different test and measurement-calibration equipment were purchased with PHARE assistance to measure surfaces (microhardness) length, profiles, contours, etc. Moreover specific equipment was purchased to test and measure the operation of brakes and brake-related equipment.

The project is in the testing phase, its positive results should be expected already in this year and from the next year on. 


\subsection{R\&D: the second Knorr-Bremse Project}

This is a strategic software development project with the participation of the Kecskemét factory of Knorr-Bremse, with its R\&D establishment in Budapest and with two dedicated R\&D institutes, the Technical University of Budapest (TUB) and the Computer and Automation Research Institute (CARI). Moreover this development is harmonized with the headquarters of KnorrBremse in Munich. This way all Hungarian developments are known and accepted in due time by the headquarters and world-wide effects can be expected as recently there are no systems in the market with parameters set in the joint $R \& D$ project..

\subsubsection{The main goals of the $R \& D$ project}

The main goals of this R\&D work is to get a new product until 1998 to assist automatically if a truck (or bus) would loose its stability or it would leave its normal, expected trace. The applied and developed sensory, software and actuator systems are based on a patented idea on asymmetrical braking.

\subsubsection{Main issues of the project}

The project started in 1995, the software development and the experimental setup will be finished in 1996, while 1997 is for the experiments and testing and for evaluation. By 1998 a tested strategic product will be available.

The main idea is to have an appropriate sensor (video camera) with appropriate software (patter recognition) to indicate when there might be problems with the stability and/or with the trace of a truck. If any irregularity is detected the brake system should be appropriately activated to compensate the problems and to avoid the loss of trace or the loss of stability. This will be done with the specifically designed, mathematically supported control system.

The applied hardware system consists of a high performance PC with a frame grabber card. A CCD camera is used for image acquisition. The PC for the lane departure detection has been connected to the data acquisition computer (already installed on the test vehicle) via serial communication interface, to acquire the front velocity and steering angle measurements needed by the detection algorithms [13]. The detection of the unintentional lane departure of road vehicles is based on the subsequent images acquired by the CCD camera and the front wheel velocity and steering angle measurements of the vehicle. The steps of image processing are the following:

1. edge detection,

2. statistical analysis of the result of the edge detection,

3. thresholding the result of the edge detection,

4. searching for lane boarders in the predicted path of the vehicle.

The method is suitable for the on-line detection in the case of clean, well-determined road surface and good light conditions. The performance of the system has been tested on different motorways in Hungary by daylight and by night. There are white markers painted on the pavement, visible very well, offering a very good field for testing the basic image processing algorithms realized by the detection system.

A fundamental adaptive algorithm has been developed on the basis of experiments on the video records made earlier with a camcorder. This algorithm worked well in the on-line experiments on the vehicle and can suit as a staring point for future refinements.

\section{Common and different features of the projects}

The PROARC COPERNICUS project was a relatively small (approx. $280 \mathrm{KECU}$ ) project still is was not a too easy task to put together the winning proposal. The Hungarian partners 
had the devotion and the necessary backgrounds and professional knowledge, however these were not enough to win. We are convinced that the knowledge of the EC requirements and of the evaluation procedures by the RWTH Aachen made this project a winning one. The work was well controlled both by the consortium itself, both by the sometimes strict review processes by the reviewers and by the EC (Brussels). We learnt our lessons including the importance of making the best possible Technical Annex to define what to do, the meetings and correspondence among the partners to define how to do, and finally to do the work together in the same level of quality, which was the guarantee of the success in both the analysis, design and implementation phases. Dissemination and practical applications are the problems of recent efforts and of the future. All partners had benefits from the joint work when we learnt the style, way of thinking, the different approaches to problemsolving, etc. of each other. These were supported with having only three partners from only two countries with no basic cultural background differences.

Based on the nature of the proposalevaluation-contract phases with their feedbacks and interactions contract subscriptions were not really fast and the project started later than could be. But when it started we did not have any real administrative problems, just the style of report-making, etc. should have been learnt.

In the case of the PHARE projects the proposals had to contain the commitment of the potential developer to cover at least $50 \%$ of all costs, the EU could offer as support only at most the other $50 \%$. As relatively high investment was planned Knorr Bremse, as a strong enterprise had real chances and could win the projects. The quality project had an overall value of approx. $100 \mathrm{KECU}$, while the R\&D project costs about $400 \mathrm{KECU}$, however for this letter project the PHARE support is only $25 \%$ of the total investment.

There was no administrative problem with the contracts with the community. Then as a next step tenders were submitted to find the best offers for the equipment needed to be purchased. The quality project was an investment supporting fund, as a matter of fact, as it supported the purchase of measurement and test equipment for a previously finished production development phase. This way there were no need to involve R\&D experts from academia.

The other project had the goal of the development of a future industrial product, but as there were some new, interesting problems to solve, the R\&D assistance of the Technical University of Budapest, and of CARI were used, as well. The researchers had to learn that the research by itself was not a goal, just a tool to achieve the goal, the new product. There are about 20 researchers taking part in this work, thus it can be stated that the PHARE support is a good tool to fight against brain-drain, too, what is a real problem in recent Hungary in the field of technical $R \& D$ and in software development. The same could be said about the COPERNICUS projects as well. The PHARE supported R\&D project is a good example of industry- academia cooperation as well, as cooperation between a smaller, local factory (Kecskemét) and the big multinational (Munich), too.

\section{Dissemination, benefits from the projects}

Recently the PROARC partners are ready to provide any appropriate robots with the communication software to have download/upload possibilities if a standard PC is available. For programming the robot and to simulate the welding besides the PROARC software the AutoCAD software is necessary, too. Moreover we are ready to add welding equipment to robots, this way providing PROARC-like arc welding solutions, however 
it is clear from this paper that further development efforts are necessary for every unique solution (inverse kinematics, program generation, maybe the communication).

The PROARC system was demonstrated in Budapest in the occasion of the final review meeting (May, 1996) where Hungarian SMEs (2-3 possible software distributors and 8-10 end users candidates) and several academic people were present. With some end users further discussions are going on. In June, 1996 the PROARC system was demonstrated in Aachen at RWTH with international participation and with a quite strong professional interest on both the software and welding sides. Discussions are going on in Germany and in Hungary with SMEs about the first real industrial applications.

If these real applications begin to work we will be able to demonstrate the results again to possible further users in all parts of Europe and we hope to have some more industrial SME users. Naturally no one should forget about the fact that our recent product is "only" a prototype and to produce working copies each time some man-months of efforts should be added for customization, etc.

As it was mentioned earlier both Knorr Bremse PHARE projects will result in the overall increase of the competitiveness of Knorr Bremse, while the results of the $R \& D$ project will be a marketable, new product running on the board computer of any trucks, where implemented..

\section{Conclusions}

The authors of this paper hope and believe that the PROARC COPERNICUS project was only a beginning for us in the $R \& D$ cooperation with Western partners under the auspices of the European Community and the lessons we learnt can be used in further international cooperations.
The same way the success of the PHARE projects are promisable to trust in the further cooperation with the European Community.

As there are several European calls for proposals (INCO COPERNICUS, ESPRIT, ESSI, etc.) where the joint participation of industry and of R\&D institutions have high priority Knorr Bremse and CARI decided to apply together for R\&D funds, with other exEastern European and with EU member industries and academia. This way every participant could find its best place in the innovation chain and stronger European products could be developed.

\section{References}

[1] Aleph Technologies: ACT Weld Offline programming software package for arc welding robots, advertisement paper, France, 1994

[2] ESPRIT PROARC No. 7831: Deliverable 2.1, Evaluation and detailed concept, 1994

[3] Booch, G.: Object-Oriented Analysis and Design with Applications, Benjamin/Cummings Inc., 1994

[4] ifp Gmbh: AnySIM-robotics, advertisement paper, Germany, 1994

[5] BYG Systems Limited: GRASP, advertisement paper, United Kingdom, 1994; The use of simulation to design and program robot welding systems, internal report, United Kingdom, 1994

[6] ESPRIT PROARC No. 7831: Deliverable 2.4, Specification of the program generation and the communication module, 1994

[7] McKerrow, P.J. Introduction to Robotics, Addison-Wesley

[8] MMS-EASE User Guide, SISCO Inc., 1994,

[9] Workspace: http://www.rwt.com/

[10] Manufacturing Message Specification, Service Definition and 
Protocol, ISO 9506-1 9506-2, EIA Standard - 511

[11] Madarász L, Holecko P, Rudas I. J.: Robotics Systems Simulations, Proc. of RAAD'96 Conf., pp. 219-222

[12] Haidegger G., Kuba R.: Highly Automated Flexible Assembly System, Proc. of Automation'95, pp. 347-353

[13] Soumelidis, A., Bokor, J. et al.: Automatic Detection of the Lane Departure of Vehicles, $8^{\text {th }}$ IFAC Symposium on Transportation Systems, Crete, Greece, 1997 